\title{
Development and Production of Modular Cosmic Ray Telescopes
}

\author{
X. He, ${ }^{a, *}$ C. Butler, ${ }^{a}$ S. Syed,${ }^{a}$ E. Potdevin, ${ }^{a}$ P. Tarrant,${ }^{a}$ N. Chen ${ }^{b}$ and T.-C. Wei ${ }^{b}$ \\ ${ }^{a}$ Georgia State University, \\ 25 Park Place, Atlanta, USA \\ ${ }^{b}$ Northwestern Polytechnical University, \\ Xian, China \\ E-mail: xhe@gsu.edu
}

While the quest of searching for the origins of the most energetic cosmic rays and the associated dynamics continues, there is a growing interest in recent years of the use of the cosmic rays for practical applications following the advancement of particle detection technologies. One of these important applications is to study the correlations between the cosmic ray flux variations and the space and earth weather at global scale in real-time at low cost. Key to these efforts is improving the understanding the correlation patterns to increase the accuracy, reliability, and timeliness of space-earth-weather forecasts. A state-of-the-art portable and modular cosmic ray muon and neutron detector prototype has been developed at Georgia State University for the measurement of cosmic ray muon and neutron flux variations simultaneously. The detector consists of three layers of plastic scintillator and a neutron-cell with liquid scintillator mounted on an extruded aluminum frame. The scintillation light is collected through embedded wavelength shifting fiber which is coupled to silicon photomultipliers (SiPM) for signal readout. The modular, portable and low cost nature of this cosmic ray telescope provides a technological choice to quantify the cosmic ray flux variation around the globe in an unprecedented spacial and time resolution. In the talk, we will highlight the details of the detector design, assembly and mass production. An initial test result will also be presented.

$37^{\text {th }}$ International Cosmic Ray Conference (ICRC 2021)

July 12 th - 23rd, 2021

Online - Berlin, Germany

\footnotetext{
*Presenter
} 


\section{Introduction}

In recent years, there are growing interests in using cosmic ray measurements for applications in space and the earth weather monitoring, non-invasive void structural tomography, soil moisture monitoring, radiation effects in health of general population, etc. [1-12] This is in part inspired by the advancement of particle detection and instrumentation over the past a few decades in acceleratorbased particle and high energy nuclear experiments. However, all of the detectors developed for cosmic ray measurement have large volumes, complex readout electronics, are expensive, and not-easy to maintain and operate. While these detectors are operated by experts uniquely at a given experimental location for their designed research objectives, it is extremely challenging if not impossible to duplicate the setup in order to perform the measurements at global scale.

Measurement of cosmic ray flux around the world in real-time is a unique approach for studying the dynamical correlation between the space/earth weather and the flux variation. This is indeed very important for understanding the forcing factors of the earth climate change. The solar activity (like Forbush events) influences geomagnetic field which in turn modulates the cosmic ray shower distribution at a given geo-position. The earth's atmosphere itself is a large particle detector in which the primary cosmic rays interact with atmospheric molecules at an altitude around $15 \mathrm{~km}$ from sea level with seasonal variations. Because of the gravitational binding, to the first order, the total air mass on earth is more or less a constant but the local air mass varies following the changes of the local barometric pressure. Measurement of the secondary cosmic ray particles reaching to the surface of the earth at a global scale in real-time is a unique tool to capture the dynamic weather patterns worldwide.

The keys for achieving the global cosmic ray measurement include, but not limited to, a few critical factors listed as follows:

- Identical detector design and performance, which eliminate the need for detector acceptance correction.

- Portability and low cost, which enable the construction and distribution of a large number of detectors worldwide in a competitive funding environment.

- Sensitivity to the changes of local meteorological parameters in shorter time frame.

- Dedicated effort of maintaining and operating the detector continuously.

Based on these requirements, a multi-layer-scintillator cosmic ray telescope have been developed at Georgia State University (GSU). The design of the scintillator tile is motivated by the excellent performance of the scintillator tiles for the sPHENIX Hadronic Calorimeter [13]. The readout consists of a Raspberry PI and a customized PCB which supplies bias-voltage to SiPMs [14] and records the data in combination of counts both from single layer and coincidences in paired-layers.

In this proceedings, a brief description of the telescope design and its components is given in Section 2 which is followed with telescope assembly and test in Section 3. A brief summary and an outlook of the future of this project are given in Section 4. 


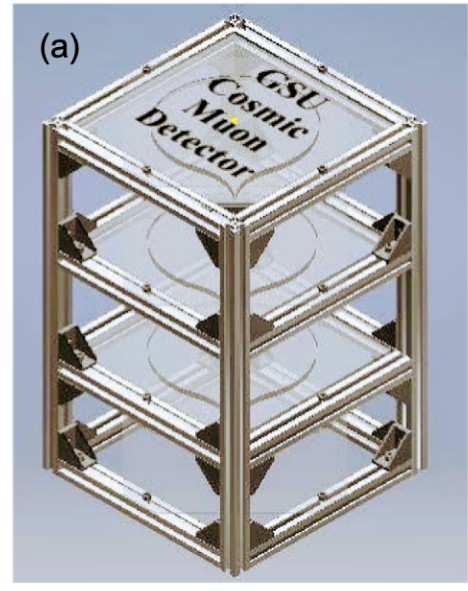

Muon telescope

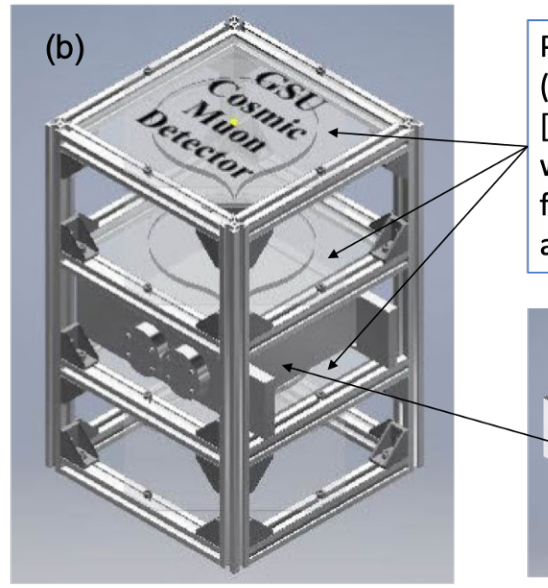

Muon/neutron telescope
Plastic scintillating panel

$(20 \mathrm{~cm} \times 20 \mathrm{~cm} \times 1 \mathrm{~cm})$

[embedded with a

wavelength shifting

fiber, SiPM is mounted

at one corner.]

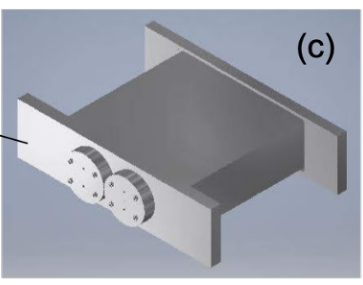

Neutron cell

Figure 1: Design of the cosmic ray telescope: (a) Muon telescope; (b) Muon and Neutron telescope; and (c) Neutron cell.

\section{Cosmic Ray Telescope Design}

Following the design goals as mentioned in the previous section, there are three generations of the cosmic ray telescope that have been developed and tested at GSU since 2016. The latest version is shown in Fig. 1. There are two types of the telescope design. One is for muon measurement only and the other with a neutron cell in order to count cosmic ray muons and neutrons simultaneously. The study of the neutron cell performance and its efficiency is still ongoing.

The key component of the telescope is the scintillator tile which not only needs to be large enough for recording statistically meaningful particle hits in a shorter time window (at about a 1-minute level) but also provides uniform scintillation light collection efficiency for hits counting. The design of the tile is based on the group's extensive work on building and testing of a Hadronic Calorimeter of the sPHENIX experiment at Brookhaven National Lab [13]. The tile dimension is $20 \mathrm{~cm} \times 20 \mathrm{~cm} \times 1 \mathrm{~cm}$ with a cutoff at a corner in order to mount a PCB board with a silicon photomultiplier (SiPM) [14]. A wavelength shifting fiber loop is glued into a shallow groove near the surface of the tile on one side. The fiber ends meet the SiPM at the corner as shown in Fig. 2. Also shown in Fig. 2 (on right) is a display of scintillation light production and propagation in the tile using GEANT4 simulation [15] in order to understand the light collection uniformity and efficiency.

The readout consists of a Raspberry PI and a custom made 8-channel readout board (the latest version). Raspberry PI provides an affordable computing and network tool for configuring the PCB and remote data taking, as shown in Fig. 3. The custom PCB interfaces with the Raspberry PI through its digital logic pins and provides controllable bias voltage, amplification and signal shaping, as well as FPGA based logic for coincidence and high speed counting. The interface to the SiPMs is made through U.FL cables providing the bias voltage and a shielded signal path for noisy environment. Another advantage of using Raspberry PI is the possibility of integrating low-cost sensors (GPS, humidity, temperature, inclination, etc.) to the telescope. The readout electronics are typically mounted on the bottom of the telescope or on its side. 

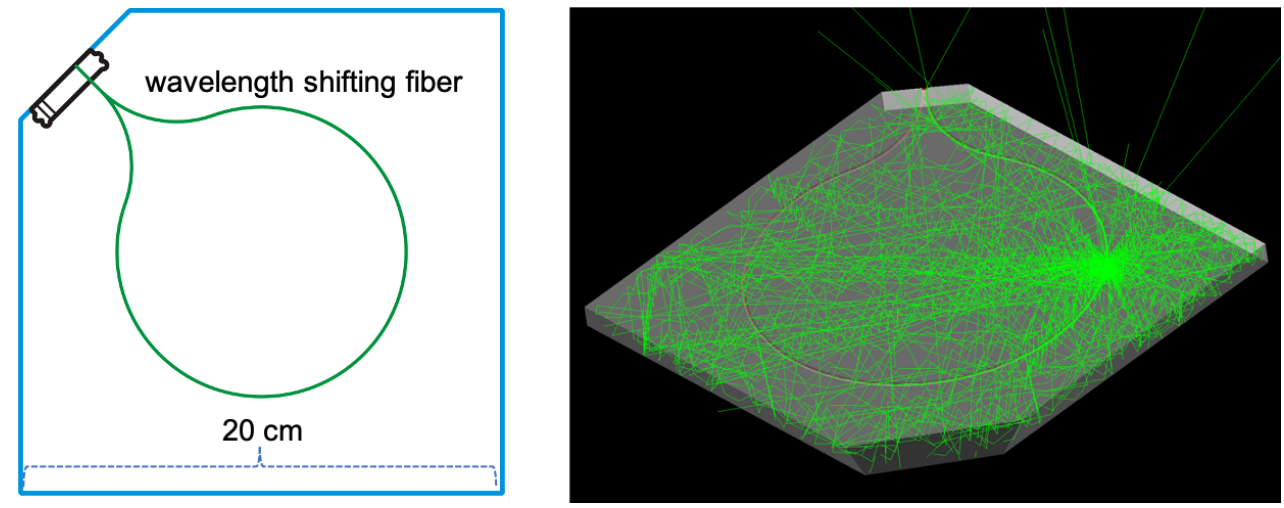

Figure 2: The design of the scintillator tile (left figure) and a display of scintillation light production and propagation inside the tile from a GEANT4-based simulation study.
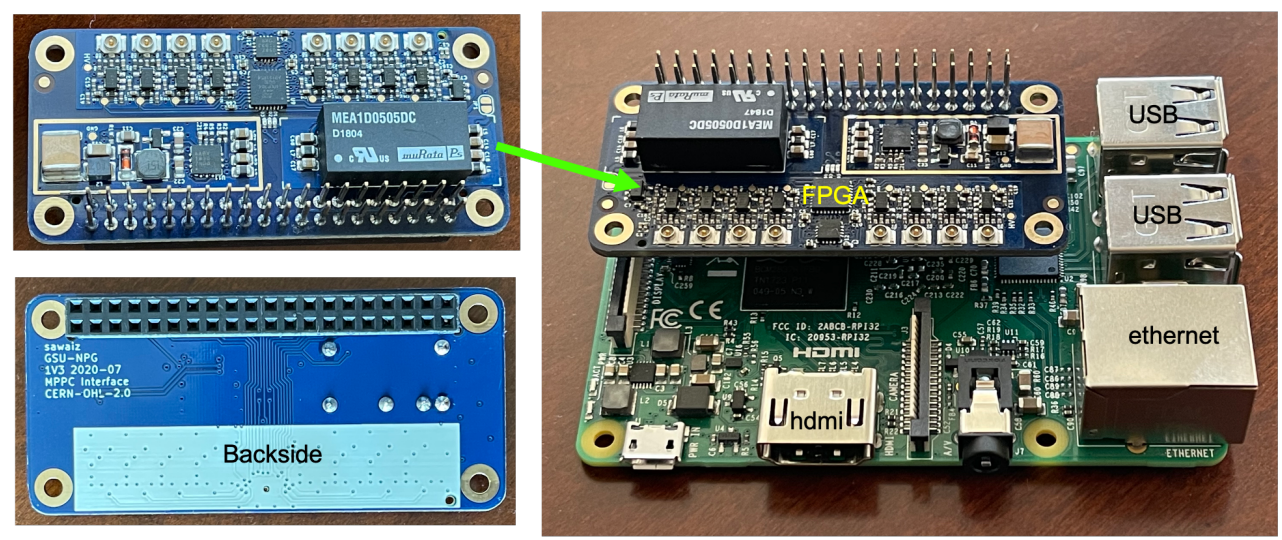

Figure 3: Raspberry PI-based readout. (upper left) 8-channel FPGA PCB; (lower left) backside of the PCB; (right) Raspberry PI with the PCB.

With the 8-channel readout, the telescope design has a greater flexibility in comparison with our previous prototypes. One can arrange as many as 8 tiles in different combination for a broader cosmic ray flux measurement with varying sky-facing angular acceptance. In our production configuration, a three-layer telescope is assembled with a $25 \mathrm{~cm}$ separation between the top and the bottom layer (see Fig. 1) while the middle layer location can be adjusted for choosing a specific angular acceptance. In order to determine the angular acceptance of a given pair of tiles, a software package has also been developed using GEANT4 with realistic material property and the exact geometry as shown in Fig. 4. One can also study the relative efficiency of muon and neutron measurement using the same software package.

\section{Telescope Assembly and Test}

The assembling and testing of the telescopes are mainly carried out by undergraduate and graduate students. Figure 5 shows two undergraduate students who were assembling telescope frames during the COVID-19 pandemic in the nuclear physics labs at GSU. We also had two high 

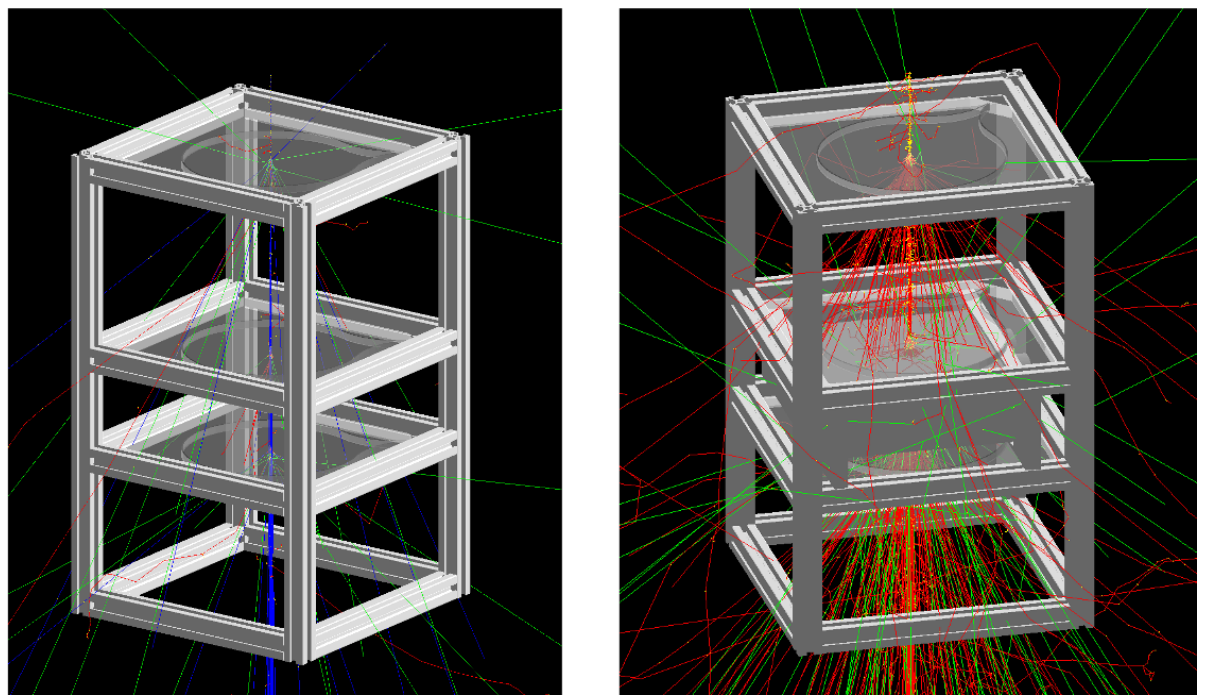

Figure 4: (color online) Acceptance study using GEANT4 simulation. (left panel) muon telescope; (right panel) muon/neutron telescope. [Red color lines represent negative charged partile tracks, blue for positive particles and green for charge-neutral particle including gammas and neutrons]
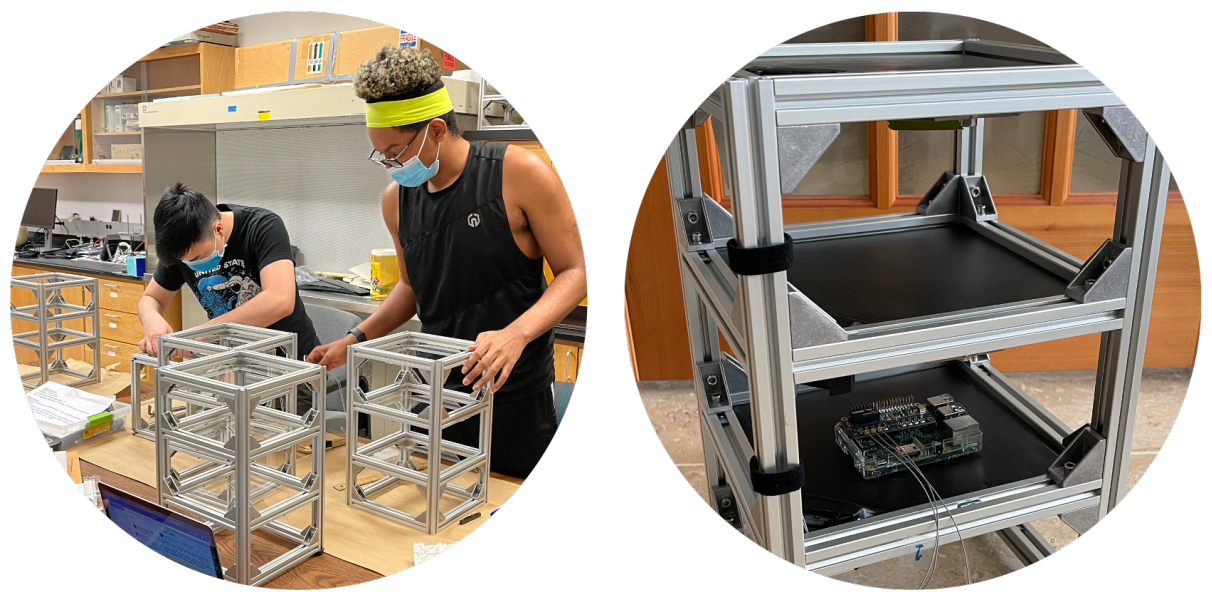

Figure 5: Two undergraduate students working on telescope assembling during COVID-19 pandemic in 2021.

school students who helped on assembling tasks during summer breaks. This project has proven to be a great tool for STEM training, which is one of the objectives of the cosmic ray project at GSU.

Our near-term goal is to assemble 50 three-layer telescopes by the end of 2021 and to make a plan for a worldwide distribution. There are close to 200 scintillator tiles made for this distribution. Half of the 200 tiles were fabricated in the physics shop at GSU while the newest tiles were made by Uniplast in Russia (the same company that produced the scintillator tiles for the sPHENIX experiment). Figure 6 shows a box of tile shipment from Uniplast (right) together with a picture of the fiber ends. Having tiles made locally provides a great opportunity for our students to learn polishing tiles and gluing wavelength shifting fibers.

Once a telescope is assembled, a multi-week test is run to study its performance and stability. 


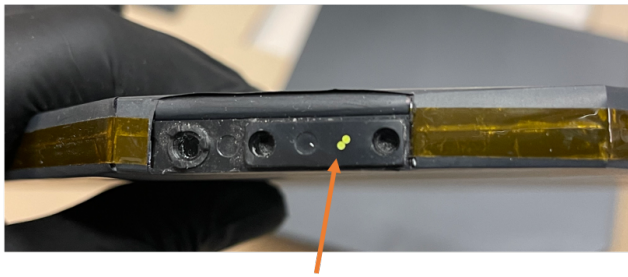

Fiber ends facing SiPM light sensor

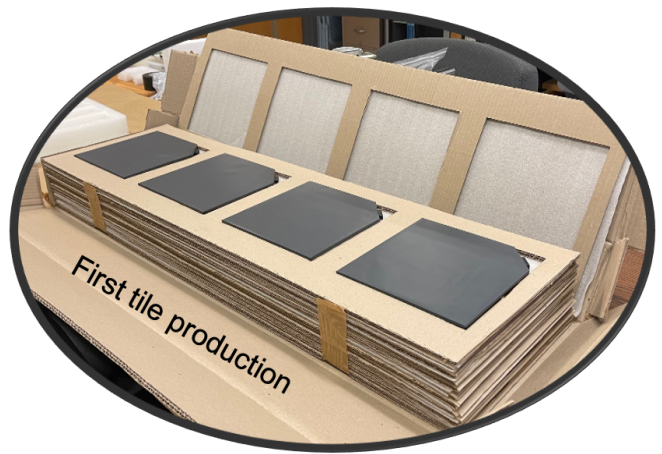

Figure 6: Scintillator tiles (ready to be installed) produced by Unplast in Russian.
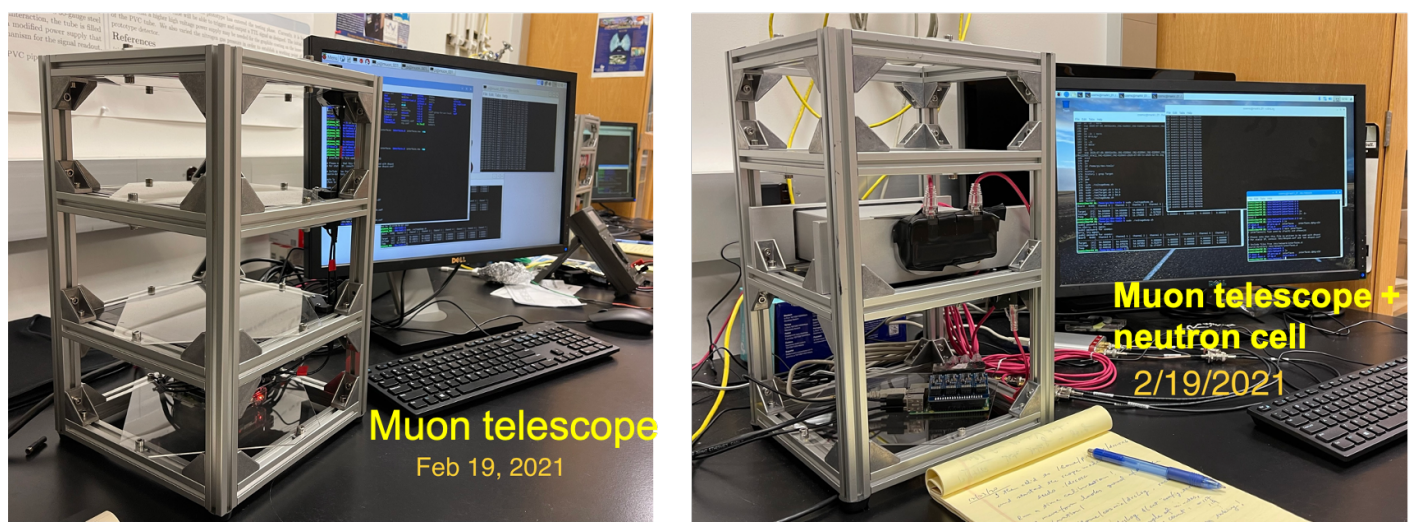

Figure 7: Test setups for monitoring the telescope performance and stability. (left) muon telescope; (right) muon/neutron telescope.

A typical test is to record a minute-long flux counts (counts from single layers and counts of coincidence from a pair of layers) $24 / 7$ and to check the sensitivity of the flux variations to the changes of the space radiation level (i.e., solar activity) and the local meteorological parameters. Figure 7 shows two such setups which have been recording data continuously for several months.

An example of the data analysis is shown in Fig. 8. The time series plots of a hourly coincidence flux in each of the paired-layer show the well-known seasonable variation of the cosmic ray muon flux reaching to the surface of the earth. Also shown in Fig. 8 is the sensitivity of the flux variation to a Forbush-like event. Arranging equal layer separation of the top-middle and the middle-bottom pair provides a self-consistent check of the telescope performance.

\section{Summary and Outlook}

We present in this proceedings an updated cosmic ray telescope design, assembly and test. The main advantages of this telescope design includes its low cost, portable nature, and flexibility for adding additional environmental sensors for broader applications of the cosmic ray measurement at a global scale. This opens up the unprecedented opportunity to built a realistic and identical cosmic ray detector network around the world for space and earth weather monitoring. 


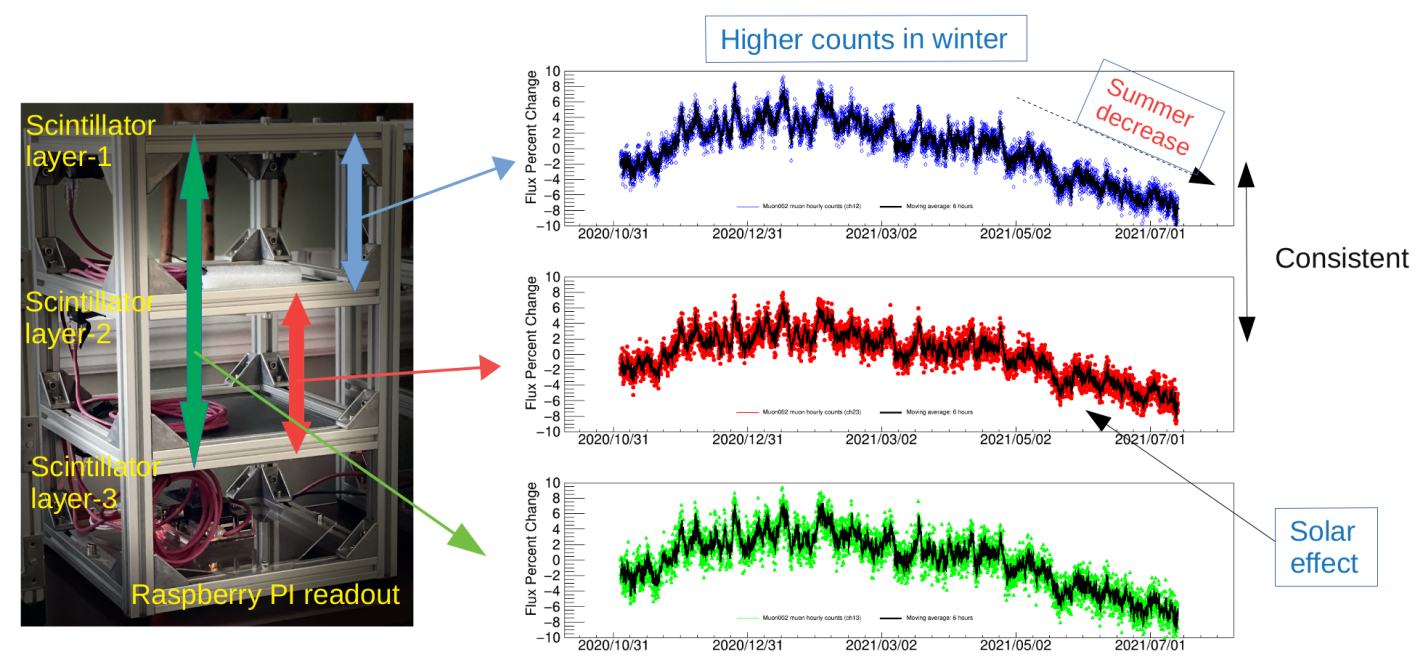

Figure 8: Example of data analysis. The top panel shows the hourly coincidence counts between the the top and the middle layer. The middle and the bottom panel show the corresponding hourly flux from the middle-bottom and top-bottom paired layers, respectively.

This project also opens up a great opportunity for STEM outreach both in regional and worldwide effort to share the learning experience beyond the age gaps and the culture difference. We are looking forward to establishing a worldwide consortium to distribute, install, and operate this network.

\section{References}

[1] Borozdin, K. N. and et al. 2003, Surveillance: Radiographic imaging with cosmic-ray muons, Nature, 422, 277.

[2] Borozdin, K. et al. 2012, Cosmic Ray Radiography of the Damaged Cores of the Fukushima Reactors, PRL, 109, 152501.

[3] Kunihiro Morishima and et al. 2017, Discovery of a big void in Khufu's Pyramid by observation of cosmic-ray muons, Nature, 552, 386-390.

[4] G. Zumerle and et al. 2018, The Cosmic Muon Tomography (CMT) Project, http:// mutomweb.pd.infn.it

[5] Jasper Kirkby, 2007, Cosmic Rays and Climate, Surv. Geophys, 28, 333-375.

[6] Lu, Q.B. 2009, Correlation between Cosmic Rays and Ozone Depletion, Phys. Rev. Lett., 102(11), 118501.

[7] A. Ollila 2012, Changes in cosmic ray fluxes improve correlation to global warming, Int. J. Phys. Scie., 7, 822-826.

[8] N. J. Shaviv 2005, On climate response to changes in the cosmic ray flux and radiative budget, J. Geophys. Res, 110, A08105. 
[9] Henrik Svensmark 1998, Influence of Cosmic Rays on Earth's Climate, Phys. Rev. Lett., 81, 5027-5030.

[10] Kennedy, A. R. 2014, Biological Effects of Space Radiation and Development of Effective Countermeasures, Life Scie. Space Res., 1, 10-43.

[11] Mertens, C. J. and et al. 2013, Space Weather, 11(10), 603-635.

[12] Wilson, J.W., \& Mertens, C.J., \& Goldhagan, P., \& Copeland, K., \& Bidasaria, H. B. 2005, Atmospheric ionizing radiation and human exposure (NASA Technical Paper, NASA/TP2005-213935, Washington, D. C.)

[13] C. A. Aidala et al., Design and Beam Test Results for the sPHENIX Electromagnetic and Hadronic Calorimeter Prototypes, in IEEE Transactions on Nuclear Science, vol. 65, no. 12, pp. 2901-2919, Dec. 2018. doi: 10.1109/TNS.2018.2879047

[14] Multi-pixel photon counter (SiPM): https://wwW.hamamatsu.com/resources/pdf/ ssd/s13360_series_kapd1052e.pdf.

[15] J. Allison, et al., Recent developments in Geant4, Nuclear Instruments and Methods in Physics Research Section A: Accelerators, Spectrometers, Detectors and Associated Equipment, Volume 835, 2016, Pages 186-225, ISSN 0168-9002, https://doi.org/10.1016/j.nima.2016.06.125. 